\title{
Tercer sector y univocidad conceptual: necesidad y elementos configuradores
}

\author{
Julio Jiménez Escobar \\ Facultad de Ciencias Económicas y Empresariales (ETEA) \\ Adscrita a la Universidad de Córdoba, España
}

\author{
Alfonso Carlos Morales Gutiérrez \\ Facultad de Ciencias Económicas y Empresariales (ETEA) \\ Adscrita a la Universidad de Córdoba, España
}

\section{Tercer sector y univocidad conceptual: necesidad y elementos configuradores}

Resumen: En el presente trabajo se destaca la importancia de que exista un concepto unívoco del tercer sector. En primer lugar se justifica esta relevancia conceptual desde un punto de vista epistemológico tanto para el estudio de los hechos sociales (objeto de conocimiento) como para la propia configuración de las comunidades científicas (sujeto del conocimiento). En segundo lugar, también se argumenta esta necesidad de univocidad ya que condiciona las diferentes políticas públicas, y porque incide en la interacción de los agentes que contribuyen al cambio social. Por último se abordan los rasgos que pueden delimitar este espacio social propio, por un lado, y a caracterizar las particularidades de las entidades que operan en el mismo, por otro.

Palabras clave: tercer sector, altruismo, economía solidaria, economía social, entidades no lucrativas.

\section{Terceiro setor e univocidade conceitual: necessidade e elementos configuradores}

Resumo: No presente trabalho destaca-se a importância de que exista um conceito unívoco do Terceiro Setor. Em primeiro lugar, justifica-se esta relevância conceitual desde um ponto de vista epistemológico, tanto para o estudo dos fatos sociais (objeto de conhecimento), quanto para a própria configuração das comunidades científicas (sujeito do conhecimento). Em segundo lugar, argumentase também essa necessidade de univocidade já que condiciona as diferentes políticas públicas, e porque incide na integração dos agentes que contribuem para a mudança social. Por último, são abordados os rasgos que podem, por um lado, delimitar este espaço social próprio, e por outro, caracterizar as particularidades das entidades que operam em ele.

Palavras-chave: terceiro setor, altruísmo, economia solidária, economia social, entidades não lucrativas, ação social.

\section{The Third Sector and Conceptual Univocality: Necessity and Essential Elements}

Abstract: This study emphasizes the importance of a univocal concept of the third sector. It first justifies the conceptual importance of univocality from a epistemological perspective and for the study of social facts (as an object of knowledge). Secondly, it argues the need for univocality since the concept is relevant to various public policies, and because it influences the integration of agents who contribute to social change. Finally, it looks at the divisions that can both confine social space and characterize the particularities of the entities that operate within it.

Key words: third sector, altruism, solidarity economics, social economy, non-profit entities, social action. 
1 Los problemas epistemológicos que genera una 'nueva realidad socioeconómica'

\subsection{Concepciones sobre el tercer sector y sus consecuencias}

La revisión de la literatura existente sobre la realidad emergente del tercer sector $^{1}$ nos muestra en su aproximación al menos dos concepciones: la anglosajona - promovida sobre todo desde Estados Unidos y la Universidad John Hopkins - y la europeacanadiense (quebequense) - promovida sobre todo desde el Centre International de Recherche et d'Information sur l'Économie Publique, Sociale et Coopérative (CIRIEC). La corriente anglosajona se posiciona desde hace ya un cuarto de siglo como la literatura del non-profit sector o del non-profit organization (entidad no lucrativa, ENL) (WEISBROD, 1975), considerando como tal a aquellas organizaciones privadas que, en virtud de sus reglas constitutivas, no pueden distribuir sus beneficios a las personas que las controlan, teniendo que destinarse aquéllos, bien a la realización de sus objetivos, bien a la ayuda de personas que no ejerzan ningún control sobre la organización. Este enfoque excluye a aquellas organizaciones que distribuyen beneficios a sus socios bajo cualquier modalidad, con lo que se descarta a la mayoría de las cooperativas como uno de las componentes del tercer sector.

Al mismo tiempo, el enfoque de ENL más divulgado (SALAMON; ANHEIER, 1992) establece cinco requisitos básicos para evidenciar a una institución como parte integrante del tercer sector (organización formal, privada, no lucrativa, con autogobierno y con participación voluntaria) entre los que no se encuentra un elemento tan característico de la visión europea como es el de la organización democrática. De acuerdo con este requisito difícilmente podrían incluirse el tercer sector a entidades tan significativas como las fundaciones o las entidades religiosas (órdenes y congregaciones religiosas, fundaciones canónicas...) que, sin embargo, sí están incluidas en el enfoque ENL.

En el ámbito europeo hay que decir que esta delimitación conceptual ya comenzó a establecerse en Francia y en Bélgica en la década de los ochenta ante la necesidad de instituir un denominador común de entidades asociadas ${ }^{2}$. Esta preocupación por las cuestiones de tipo conceptual estuvo en el origen del primer gran grupo de trabajo internacional que el CIRIEC puso en marcha en 1988, y cuyos resultados fueron publicados en forma de libro, en sendas ediciones, española y francesa (MONZÓN; DEFOURNY, 1992; MONZÓN; DEFOURNY, 1992), alcanzando una notable difusión. Los criterios utilizados por dicho grupo fueron acordes con la tradición de los principios cooperativos: carácter mutual ${ }^{3}$ (finalidad de servicio a la colectividad antes que de lucro), autonomía e independencia de poderes públicos o privados, gestión democrática, primacía de las personas y del trabajo sobre el capital en el reparto de las rentas.

Durante toda la década de los noventa han proseguido los esfuerzos por encontrar elementos de convergencia entre la concepción europea y la anglosajona. Ya a finales de la década de los ochenta, la Comisión Científica del CIRIEC-España propuso un nuevo enfoque denominado de economía social, integrador de la concepción tradicional y del enfoque ENL que se apoyaba en los principios cooperativos y en la metodología de la contabilidad nacional, e identificaba en el sector de mercado de la economía social a las empresas con organización democrática (una persona, un voto) y con distribución de beneficios no vinculada al capital aportado por el socio, incluyendo en el sector de no mercado de la economía social a las instituciones privadas sin fines de lucro (BAREA, 1990) ${ }^{4}$.

No cabe duda de que la delimitación conceptual del tercer sector continúa siendo un tema abierto al debate y dista mucho de estar resuelto presentando todavía unos perfiles borrosos y no suficientemente definidos. Sin embargo, el identificar adecuadamente los elementos y caracteres que lo definen tiene una importante significación, como pondremos de manifiesto en el siguiente apartado.

\subsection{Necesidad de avanzar en la construcción de un concepto unívoco}

Consideramos que cualquier análisis sobre la nueva realidad socioeconómica que supone el tercer sector y sus perspectivas de evolución, así como sobre el papel que puede desempeñar en el contexto de un sistema económico plural para contribuir a resolver algunos de los problemas económicos más relevantes de nuestro tiempo precisa avanzar en la construcción de un término que, de forma unívoca, designe una determinada realidad social y económica. Esta afirmación se sustenta en las razones que seguidamente se exponen.

\subsubsection{Determinación de los hechos científicos, técnicas a emplear y expectativas de resultados}

El que exista un concepto unívoco que describa la realidad que constituye el tercer sector resulta necesario desde una perspectiva epistemológica por diversos motivos. En primer lugar, porque el concepto contribuirá a delimitar adecuadamente la realidad que debe ser objeto de estudio por parte de la comunidad científica ${ }^{5}$. Con ello se evitará que la atención se disperse en realidades próximas, pero que no presentan una homogeneidad similar, contribuyendo con ello a centrar los esfuerzos de las comunidades científicas. 
En segundo lugar, ayudará a definir las prácticas científicas adecuadas para describir y explicar esta realidad, ya sean de tipo cuantitativo o cualitativo. Al mismo tiempo permite identificar los problemas que se plantean, tanto en el ámbito de esa realidad socioeconómica previamente acotada, como en sus interrelaciones con el resto del entramado socioeconómico. Con todo ello se podrá comprender y explicar de forma más precisa la realidad objeto de estudio, lo que constituye, en última instancia, el objeto de cualquier actividad científica.

\subsubsection{Relevancia para determinar las comunidades científicas implicadas}

Pero contar con una realidad social acotada y conceptualizada no sólo delimita el objeto de análisis, las técnicas a emplear para su estudio, y los problemas relevantes, sino que indirectamente concreta también el ámbito de los sujetos llamados a investigar esa realidad (las comunidades científicas). Esto es así porque las comunidades científicas, en última instancia, se constituyen en torno a un objeto de investigación, unas prácticas científicas, y unas expectativas de resultados, que se suelen enmarcar todas ellas en unos determinados marcos teóricos ${ }^{6}$. Sólo a partir de que las comunidades científicas hayan tomado, desde un punto de vista sociológico, conciencias de sí mismas, podrá avanzarse solidamente en la construcción de estructuras administrativas que contribuyan a su consolidación. Estas es una de las razones principales por las que la economía social, en cuanto que disciplina que estudia el tercer sector, no suele estar reconocida administrativamente como área de conocimiento -junto con la necesidad de un abordaje en muchas ocasiones interdisciplinario -, por lo que tampoco tiene posibilidad alguna de formar parte como tal de los Departamentos Universitarios?

Pero si las estructuras académicas son necesarias, tan importante es también la generación de espacios de interlocución entre la sociedad - con sus problemas y sus innovaciones sociales -, la universidad - con su rigor metodológico y sus propuestas - y la economía social - con sus realidades en movimiento. En ciencias sociales el investigador debe saber orientarse adecuadamente en la realidad social que trata de explicar, pues sólo así podrá captar y comprender el verdadero alcance de las situaciones y problemas que se plantean, para explicarlos a través de sus conceptos teóricos ${ }^{8}$. Este espacio está por hacer. Los lugares de encuentro son insuficientes para generar el clima, profundizar en los problemas y establecer líneas de avance.

\subsubsection{Significación para los avances que supone el tercer sector}

Todo lo que venimos exponiendo tiene una importancia añadida en el ámbito de las Ciencias Sociales, pues como señala Adorno (1973, p. 121138) la sociedad, a diferencia del mundo de la naturaleza que, en cierta medida, nos viene dado en sus "estructuras fundamentales", es en potencia mucho más de lo que es, por lo que se pueden generar estructuras económicas, jurídicas y sociales diferentes a las existentes. Pero es que además todas estas estructuras tienen una gran relevancia en la actuación de los sujetos y, por ende, en la generación de nuevas estructuras o la perpetuación de las existentes. En efecto, como revela Giddens (1997, p. 193) "la estructura es constituida por una acción, y recíprocamente [...] la acción está constituida estructuralmente". Es decir, las acciones de los agentes generan determinadas estructuras, pero, a su vez, aquellas acciones están condicionas por las estructuras existentes en las que se enmarcan 9 . Estas estructuras no simplemente constriñen el obrar humano, sino que son también habilitadoras ${ }^{10}$. Por ello, la explicitación de las estructuras económicas, jurídicas y sociales, su análisis crítico y sus propuestas de mejora resultan de gran relevancia para el investigador en ciencias sociales.

Para la creación de estas estructuras cobran gran importancia las políticas públicas, ya sea generando actuaciones legislativas que creen determinados marcos jurídicos de procedimiento, ya en la propia planificación e intervención de los poderes públicos a través de medidas de fomento y apoyo que orientan las actuaciones de los agentes sociales, recurriendo a medidas que suelen tener un reflejo presupuestario (subvenciones y ayudas). Todas estas actuaciones de los poderes públicos se enmarcan no sólo en consideraciones de tipo político, sino también en determinados marcos teóricos elaborados por las comunidades científicas, que obran como presupuestos 
partir de los cuales las autoridades públicas adoptan sus decisiones, sobre todo en el ámbito de las políticas económicas, ya sean generales o sectoriales, $\mathrm{y}$ de las políticas legislativas. No pretendemos con estas afirmaciones dar más relevancia de la necesaria a los resultados de las investigaciones en ciencias sociales, pero tampoco ignorar su importante influencia en el diseño de las políticas públicas.

También las actuaciones de los agentes sociales se verán influidas dependido de la concepción, explícita o implícita, que tengan de la realidad en la que operan. En esta línea, las técnicas de gestión a emplear en las entidades del tercer sector dependerán mucho de la concepción que se tenga de las funciones sociales de estas entidades y de su estructura organizativa ${ }^{11}$.Por todo lo expuesto, consideramos de gran importancia ofrecer una serie de elementos que contribuyan a delimitar el espacio social que podemos definir como tercer sector, por un lado, y los rasgos específicos de las entidades que actúan en dicho espacio, por otro.

\section{La delimitación de un espacio social 'propio'}

\subsection{Escenarios sociales}

Tradicionalmente, la actividad económica y social ha estado delimitada por el protagonismo fundamental de dos sectores: el público y el privado. Sin embargo, en las últimas décadas del siglo XX se ha ido configurando y resaltando una realidad emergente que no se corresponde con los estereotipos que se deducen de aquella concepción bipolar de la realidad social que recogía la presencia de un interés público y privado. En este contexto, la denominación de tercer sector fue empleada originariamente por Levitt (1973, p. 48-49), designándose con ella un conjunto complejo y heterogéneo de entidades que suelen definirse por exclusión: no son públicas, pero tampoco tienen fines lucrativos (CABRA DE LUNA, 1998, p. 24).

Esta nomenclatura utiliza una clave interpretativa que se basa en la distribución tripartita del espacio social entre diversas instituciones básicas: el poder, el dinero y la solidaridad (GARCIA ROCA, 1996, p.11) ${ }^{12}$. Desde esta comprensión de la realidad social ${ }^{13}$, cada escenario responde a una lógica distinta: la del derecho, la del intercambio y la del don (GARCÍA ROCA, 1996, p. 24-25). La primera de ellas opera en el escenario del Estado creando los espacios administrados. En el escenario del derecho, la persona es ante todo un ciudadano. Sus relaciones con el poder público aparecen siempre normadas por el Derecho, que se encarga de regular los derechos individuales, civiles y sociales. Es un mundo reglado, con todo lo que esto comporta: derechos y deberes exigibles, procedimientos articulados al efecto y 'burocracia' para su aplicación (administración y poder judicial). Es un mundo burocratizado y, por ello, poco flexible. Las relaciones entre sujetos, funcionarios y ciudadanos están relativamente 'cosificadas'.

La lógica del intercambio se sustancia en el escenario del mercado, que se estructura sobre la negociación contractual y se regula por acuerdos o convenios. En este escenario, la persona es primariamente comprador o vendedor (GARCÍA ROCA, 1996, p. 27). Es un mundo en el que prima la libertad - la autonomía de la voluntad -, sometida a ciertas reglas. La actuación como operador en el mercado exige el intercambio de prestaciones, quedando al margen del mismo quien no tiene nada que intercambiar. Es un mundo mercantilizado, muy flexible para quien es solvente - tiene bienes que intercambiar - y absolutamente inflexible para quien no lo es: ni siquiera puede acceder al mismo. Pero, para el que es solvente, la lógica del intercambio es una lógica también relativamente 'cosificada', pues en la misma, interpersonalmente hablando, sólo actúa la dimensión negociadora de la persona, con todo lo que la misma comporta: apariencia de fortaleza, intento de ganar en la negociación, competitividad etc.

Finalmente, hay otra esfera social en la que las relaciones interpersonales operan con 'mayor intensidad' y en la que actúa la solidaridad. Son lugares donde sigue viva la exigencia de sentido y de comunicación interpersonal. Esta lógica del don es muy adecuada para producir y distribuir ciertos bienes sociales que se caracterizan, precisamente, por la proximidad, la comunicación y la personalización (GARCÍA ROCA, 1996, p. 24-25) ${ }^{14}$.

\subsection{Funciones del tercer sector}

\subsubsection{Integración de agentes solidarios}

No obstante, resulta indiscutible que es en el último escenario descrito en el que el valor de la solidaridad puede encontrar un espacio más amplio, ya que, por una parte, la lógica del intercambio dificulta e imposibilita, en gran medida, la presencia de este valor ${ }^{15} \mathrm{y}$, por otra, la excesiva burocratización y reglamentación establecida desde arriba (ámbito del derecho) imposibilita que el sentimiento de solidaridad encuentre el cauce que necesita: la cercanía para la percepción y asunción del problema ajeno y la libertad necesaria para actuar conforme requiere la situación concreta ${ }^{16}$.

Es en este espacio en el que la solidaridad social puede encontrar una dimensión cualitativamente diferente, en el que actúan un elenco heterogéneo de entidades y organizaciones que podemos afirmar que constituyen, cada una de ellas en sí misma, un cauce 
institucionalizado para la expresión de la solidaridad voluntaria de multitud de agentes sociales: los promotores de estas entidades (el fundador en las fundaciones y socios promotores en las asociaciones), los cargos directivos o 'consejeros' (los patronos en las fundaciones o los miembros del órgano de representación en las asociaciones $)^{17}$, los que colaboran gratuitamente con estas entidades, ya sea con aportaciones de contenido económico - mecenas - o con prestaciones de carácter personal voluntarios. Incluso, en el caso de que exista una retribución - supuesto de los asalariados o, en algunos casos, los 'consejeros' -, la misma no tiene por qué desnaturalizar la componente solidaria del trabajo desempeñado, que puede estar cargado de una significación y alcance diferentes a los que tienen en otras entidades de carácter mercantil ${ }^{18}$. Desde esta perspectiva, las ENL aglutinan las motivaciones de sujetos que presentan inclinaciones de este tipo, impidiendo con ello que se pierdan por falta de cauces de expresión. En este sentido cumplen una función integradora de sujetos con motivaciones de carácter solidario (JARRE, 1991).

\subsubsection{Función axiológica}

El espacio social del tercer sector representado por las ENL genera un valor añadido que se proyecta ad extra de las propias entidades, puesto que aportan valores y servicios a la sociedad distintos de los del Estado y el sector privado mercantil.

Por una parte, realiza una función de tipo axiológico o de promoción de determinados principios y valores en el contexto social: los valores de la participación, la solidaridad, la responsabilidad social y el pluralismo. En este sentido, las entidades sin fin de lucro no sólo contribuyen a que no se pierdan las tendencias solidarias ya existentes en muchas personas, sino que, además, en multitud de ocasiones, y a través de la participación de estos sujetos en las mismas, las desarrollan y fortalecen. Asimismo, suscitan y despiertan inclinaciones solidarias en otros muchos agentes sociales que hasta ese momento no las habían desarrollado ${ }^{19}$. Esta función cobra todavía más relevancia en un contexto en el que los valores del mercado invaden todos los terrenos de la vida social ${ }^{20}$.

\subsubsection{Promoción de la innovación y el cambio social}

Por otra parte, estas entidades realizan ciertas tareas sociales para las que las administraciones públicas y las empresas lucrativas están en gran medida incapacitadas, ya sea por su lógica funcional o por su nivel de implicación en el sistema social. Nos estamos refiriendo a la labor 'explicitadora', por un lado, y a la labor de denuncia y reivindicación, por otro. La primera tiene que ver con la gran capacidad que muestran las ENL para percibir y explicitar nuevas situaciones sociales o necesidades de grupos específicos o de pueblos enteros, en definitiva, innovar socialmente (JARRE, 1991). Esta tarea explicitadora está relacionada, también, a su especial idoneidad para hacer "aflorar a la conciencia colectiva necesidades sociales hasta entonces ignoradas o mal conocidas ${ }^{21}$." Las estructuras burocráticas - jerarquizadas y poco flexibles - de las administraciones públicas las merman considerablemente para esta labor. La atención polarizada casi de modo exclusivo en las realidades sociales que pueden suponer una oportunidad de negocio, y que por ello requieren una demanda solvente, limita enormemente la forma de mirar a la sociedad de las entidades mercantiles. Estas dos 'servidumbres' no aparecen en las ENL: no miran a las realidades sociales con el objeto de obtener un beneficio, ni se aproximarán a las mismas desde unas estructuras rígidas y burocráticas, sino desde una lógica organizativa más ágil y flexible 22 .

Pero las ENL no sólo descubren nuevas realidades sociales, sino que, una vez descubiertas, por ellas mismas o por otros agentes sociales, son las que mejor pueden realizar una labor de denuncia y reivindicación, ya que las administraciones públicas y las empresas presentan una mayor implicación con el statu quo imperante en cada momento (función profética).

\subsubsection{Generación de servicios con caracteres singulares y de capital social}

Existen otras ocasiones en las que las ENL concurren en la oferta con entidades públicas o mercantiles, pero con unos matices y significaciones muy diferentes respecto a los servicios que ofrecen. La concurrencia de las entidades del tercer sector se produce, fundamentalmente, en el ámbito de los servicios (asistencia social, educación, sanidad, investigación), y no en la producción de bienes, operando por ello una serie de características que son inherentes a cualquier prestación de servicios, y que en este contexto adquieren una especial relevancia, pues ello es lo que permite que la oferta de las ENL pueda cobrar una significación muy diferente. Estas características de los servicios tienen incidencia en la producción, consumo y valoración o evaluación de su calidad ${ }^{23}$. Todas las características inherentes a los servicios provoca que en su prestación puedan cobrar gran relevancia las interrelaciones - y no sólo económicas - entre prestador y receptor: al implicarse en la propia producción del servicio su usuario, al ser la producción y el consumo simultáneo, y al depender la evaluación de la calidad del servicio de numerosos intangibles vinculados, en gran medida, a la relación entre oferentes y demandantes del servicio. $\mathrm{Y}$ es en este mundo de interrelaciones y encuentros personales en el que las entidades del tercer sector pueden actuar de una manera significativamente dis- 
tinta (BEN-NER; GUI, 2002). El carácter prevalente del fin o misión de la entidad del tercer sector - en las que no se persigue un lucro partible, a diferencia de las entidades mercantiles - y el mayor ámbito de libertad en lo que se refiere a sus actuaciones - a diferencia de las relaciones administrativas - es lo que permite marcos más apropiados para esas relaciones de encuentro e interrelación, en los que se puede generar una comunidad y un universo de significaciones con carácter propio y que resulta muy relevante en las demandas de servicios en las que la persona que los requiere se siente, de alguna forma, en una situación de 'debilidad' o 'inferioridad' (asistencia social, sanidad, educación), mostrando con ella una mayor necesidad de comprensión y cercanía, en tanto que está mostrando un aspecto de su persona que transciende de lo que constituye un mero intercambio de prestaciones con valor económico ${ }^{24}$. Todas estas circunstancias son las que nos permiten afirmar que las entidades públicas se relacionan con ciudadanos, las empresas mercantiles lo hacen con sus clientes y las ENL, con sus beneficiarios, en cuanto lo fundamental de la relación no es el mero intercambio - relación vendedor-cliente -, sino que se requiere un grado de implicación mucho mayor, sin que esto suponga negar que la relación de intercambio también éste presente, aunque de manera diferente, en las ENL ${ }^{25}$.

Desde esta perspectiva, las ENL cumplen una importantísima función en la prestación de servicios relacionales y son un cauce privilegiado para generar capital social ${ }^{26}$ en la medida que potencian la relación personal y las redes sociales que contribuyen a la generación de acciones colectivas en beneficio de la propia comunidad.

\section{La especificidad de los agentes sociales: predominio de la motivación intrínseca}

El tercer sector, a la vista de lo desarrollado anteriormente, se puede definir como el espacio social que forman un conjunto heterogéneo de entidades que tienen como finalidad realizar funciones de denuncia y reivindicación social (función profética) o prestar servicios en los que prime una relación de encuentro con el beneficiario (función de producción de servicios relacionales), que contribuyen a garantizar y promover determinados valores sociales (función axiológica), y que constituyen, por todo ello, un cauce institucionalizado para el ejercicio de la solidaridad voluntaria de muchos ciudadanos (función integradora).

De dichas funciones sociales se derivan una serie de consecuencias en el ámbito relacional: existen motivaciones diferentes en los sujetos para implicarse en una ENL que en una entidad lucrativa. En las en- tidades lucrativas las principales motivaciones de los sujetos que forman parte de la organización tienen que ver con el deseo de obtener un lucro o ganancia económica, sin perjuicio de que también puedan estar presentes otras aspiraciones: la vocación, la integración social, el desarrollo a través del trabajo. Por otra parte, las relaciones entre el oferente y el demandante se fundamentan en una relación de intercambio entre el valor económico de las prestaciones. La ganancia y el valor de cada prestación son 'bienes' tangibles que pueden ser objeto de medición en términos monetarios.

En las ENL el altruismo es lo que generalmente mueve a los promotores de la entidad, a los 'consejeros', a los donantes (mecenas) y a los voluntarios. También será frecuente, y necesario al menos en un porcentaje importante de los mismos, que los directivos y trabajadores no busquen exclusivamente una gratificación económica, sino que sintonicen en forma significativa con la misión, objetivos, valores y cultura organizativa de la entidad. Estas motivaciones diferentes tienen a su vez otras consecuencias en diferentes aspectos de la organización que seguidamente destacamos.

\subsection{De la hegemonía de lo tangible y cuantitativo a la importancia de lo intangible y cualitativo (valores)}

Las variables cualitativas cobran una mayor relevancia en las ENL tanto para los miembros y colaboradores de la organización como para los beneficiarios de sus actuaciones. En efecto, en las entidades lucrativas el grado de satisfacción de sus componentes se puede medir fácilmente en términos cuantitativos - monetarios - dado que lo que esperan es la obtención de una ganancia: dividendos (los accionistas), intereses (los prestamistas) o salario (los consejeros, directivos y trabajadores). En cambio, en las ENL lo que los sujetos implicados esperan, en mayor o menor medida, es otro tipo de 'gratificaciones' de carácter más cualitativo e intangible. Todo ello conlleva a que en la planificación de las actividades a realizar y en la forma de llevarlas a cabo deban estar presentes aquellos intangibles, y que en la evaluación de las mismas no juegue como indicador fundamental la obtención del beneficio económico generado. Esto se concreta (HERRERA GÓMEZ, 1998, p. 170) en que las decisiones que se tomen se encaminen a asegurar la elaboración del mejor servicio posible en función de los recursos disponibles - no se busca la maximización del beneficio - y en que el éxito no se mida en términos de incremento de beneficios sino por el número de servicios producidos y el modo de hacerlo.

Los clientes de las entidades mercantiles desean, esencialmente, realizar un intercambio ventajoso o, al menos equivalente, en cuanto al valor económico de 
las respectivas prestaciones: servicio prestado y precio pagado. En cambio, los beneficiarios de los servicios de las ENL esperan, en muchas ocasiones, no sólo un intercambio equivalente o ventajoso económicamente hablando - esto en los supuestos en que haya precio -, sino también un servicio prestado de forma cualitativamente diferente que posibilite la relación de 'encuentro' personal a la que aludíamos en el epígrafe segundo. Y en esta relación es indiscutible que intervienen muchas variables cualitativas.

\subsection{De la importancia de los shareholders (propietarios) a la relevancia de los stakeholders (implicados-partícipes)}

Las entidades lucrativas o mercantiles nacen para generar una riqueza que va a ser objeto de reparto entre los distintos sujetos que colaboran en su producción: socios capitalistas, consejeros, directivos, trabajadores y financiadores externos (entidades financieras y obligacionistas). Para crear esa riqueza la entidad debe realizar una serie de actividades productivas (el objeto social) que de esta forma adquieren un cierto carácter instrumental. Los sujetos implicados en la organización satisfacen esencialmente sus expectativas si en términos económicos consideran gratificadas sus aportaciones (capital o trabajo a la entidad), siempre que, por supuesto, la entidad consiga sus resultados económicos a través de la realización de una serie de actividades en el marco de la legalidad y moralidad vigentes. Pero el principal elemento motivador para los sujetos implicados en la organización no es la actividad en sí misma, sino la generación de una riqueza que se distribuye entre los distintos componentes de la organización ${ }^{27}$. Esto es lo que lleva a algunos a afirmar que se pueden separar, relativamente, el diseño de las actividades a realizar y la evaluación de las mismas - labor que competería casi en exclusiva a los consejeros y directivos de la organización - de la satisfacción de los intereses de trabajadores y financiadores, lo que se consigue gratificando en términos económicos sus aportaciones al proceso productivo.

Por el contrario, las ENL se caracterizan por tener una misión que se convierte en su razón de ser cohesionando las motivaciones y expectativas de los distintos colectivos que se integran en ella. Por ello, la percepción de que dicha misión se está cumpliendo adecuadamente es determinante para la cohesión interna y la pervivencia de la entidad: constituye el principal elemento incentivador para que varios colectivos (promotores, consejeros, directivos, empleados, donantes y voluntarios) dediquen sus ‘energías' a la organización. En este sentido, la misión tiene un carácter ilusionante, y para que ésta ilusión se produzca, los distintos 'factores productivos' no sólo deben ser combinados adecuadamente 'desde arriba' (la dirección de la entidad) sino que tienen que tener una mayor implicación y percepción respecto a lo que la entidad realiza hacia fuera (el qué se hace y cómo se hace), pues esto es lo que les lleva, fundamentalmente, a implicarse en ella. Desde esta perspectiva, los miembros de estas organizaciones se ven mucho más llamados a participar en los procesos de planificación, decisión y evaluación de las actividades. De esta forma, cumplimiento de la misión y satisfacción de los miembros de la organización irán normalmente parejos.

El cauce institucionalizado para el ejercicio de la solidaridad que suponen las ENL lleva a que haya sujetos que lo utilicen para desarrollar sus sentimientos de altruismo y solidaridad. Este es el caso de los que colaboran financieramente con sus donativos (particulares, empresas, obra social de las cajas de ahorros) o con su trabajo gratuito (voluntarios). Esta particularidad introduce ciertas especificidades en la gestión de las ENL: por una parte puede suponer una mayor riqueza, en cuanto que la entidad puede contar con más colaboradores, pero también comporta una mayor complejidad.

\subsection{De unas relaciones bilaterales a unas relaciones unilaterales}

En muchas de las relaciones, sobre todo a nivel interno, que se entablan con las ENL no existe una gratificación económica por los bienes entregados (mecenazgo) o servicios prestados (voluntariado). Este contexto económico asimétrico de la relación entre los agentes lo denominamos ausencia de bilateralidad o unilateridad. El mismo comporta, entre otras consecuencias, que los compromisos que se contraen con la organización por parte de los donantes o voluntarios no sean muy estables al no estar formalizados contractualmente.

La unilateridad también conlleva que, en ocasiones, la entidad se pueda ver tentada a satisfacer, en mayor medida, a aquellos de quienes depende financieramente-los mecenas - que a aquellos que justifican fundamentalmente su existencia: los beneficiarios. La ausencia de equivalencia entre el valor económico del servicio prestado y el precio que por el mismo paga el beneficiario - si es que lo hay - puede provocar el efecto anterior ${ }^{28}$. En otros términos, la ausencia de bilateralidad económica genera un marco más 'difuso' para la adecuada valoración de los output o servicios que presta la entidad, en tanto que el que disfruta de ellos no es del que depende financieramente hablando la organización. En todo caso, aún siendo consciente de este peligro y evitándolo, es evidente que la gestión de este tipo de organizaciones se complica, pues la calidad del servicio deberá satisfacer, simultáneamente, y, en gran medida, tanto a los 
beneficiarios como a los financiadores y, en general, a los colaboradores altruistas de la entidad.

\subsection{De las oportunidades de negocio al cumplimiento de la misión: menores alternativas pero más profundidad de actuación estratégica}

Las entidades lucrativas, al pretender como fin último obtener una ganancia económica que será objeto de un ulterior reparto entre sus miembros, cuentan con una considerable libertad de actuación en la elección de los medios para alcanzar tal objetivo: pueden diversificar enormemente la gama de actividades a realizar, abandonar sin muchos problemas aquellas que no resulten rentables, adaptarse a las preferencias y gustos del mercado en cada momento etc. Por el contrario, el carácter misionero de las ENL establece importantes limitaciones en relación con las actividades a emprender (HERRERA GÓMEZ, 1998, p. 185) e, incluso, con respecto a la forma de llevarlas a cabo ${ }^{29}$. Para llevar a cabo la misión son idóneas unas actividades y no otras: no se podrá dar prioridad a las que no sean adecuadas para su cumplimiento. Soslayar esta idoneidad puede originar problemas legales y fiscales, pero, sobre todo, supondría 'dinamitar' a la propia entidad, ya que la misión es la que cohesiona las diversas motivaciones y expectativas de los distintos colectivos integrantes.

Todos estos aspectos que hemos señalado definen un nuevo paradigma organizativo que se adapta a un contexto organizativo más ambiguo - dada la relevancia de los intangibles y de la exigencia de una mayor implicación de los agentes -, más complejo existencia de sujetos específicos y peculiares - e inestable respecto a los compromisos asumidos con la organización - derivado de la especificidad en los sujetos implicados junto con la unilateralidad. Además, de la unilateralidad y el reducido espectro de actuaciones puede derivarse la exigencia de una mayor pertinencia de las acciones emprendidas: deben ser aptas y adecuadas para plasmar en la práctica el cumplimiento de la misión institucional. Por último, de las menores alternativas de actuación y de la importancia de los intangibles se deduciría la necesidad de que, para que la misión se cumpla, las actuaciones que se realicen internamente se hagan de modo que satisfaga, en gran medida, a los miembros de la organización, tanto en el proceso de prestación del servicio como en la obtención de resultados.

\section{Referencias}

ADORNO, T. W. Sobre la lógica de las ciencias sociales. In: et al. La disputa del positivismo en la sociología alemana. Barcelona: Edit. Grijalbo, 1973.
BALLESTERO, E. Economía social y empresas cooperativas. Madrid: Alianza Universidad, 1990.

BAREA, J. Concepto y agentes de la economía social. CIRIEC-España, n. 8, p. 109-118, 1990.

BEN-NER, A.; GUI, B. The Theory of Nonprofit Organizations Revisited. In: ANHEIER, H. K.; A. BEN-NER. Advances in Theories of the Nonprofit Sector. London: Kluwer/Plenum Books, 2002.

CABRADE LUNA, M. A. El tercer sectory las fundaciones de España hacia el nuevo milenio. Enfoque económico, sociológico y jurídico. Madrid: Ed. Escuela Libre Editorial, 1998.

CHAVES, R. et al. Economía social y empleo en la Unión Europea. Valencia: CIRIEC, 2000.

DEFOURNY, J.; MONZÓN, J. L. Economie sociale, entre economie capitaliste et economie publique; The Third Sector, Cooperative, Mutual and Nonprofit Organizations. Bruxelles: Ed. De Boeck - Wesmael, 1992.

FUNES, M. J. Altruismo. In: ARIÑO, A. Diccionario de la solidaridad(I). Valencia: Ed. Tirant lo Blanc, 2003, p. 21-28.

GARCÍA DELGADO (Dir.). Las cuentas de la economía social. Madrid: Thomson-Civitas, 2004. Dir.

GARCÍA ROCA, J. El tercer sector. Revista de Documentación Social, Caritas Española, n. 103, p. 11-35, 1996.

GIDDENS, A. Las nuevas reglas del método sociológico. Buenos Aires: Ed. Amorrortu Editores, 1997.

HERRERA GÓMEZ, M. La especificidad organizativa del tercer sector: tipos y dinámicas. Papers, Revista de sociologia, Universidad Autónoma de Barcelona, n. 56, p.163-196, 1998.

JARRE, D. La iniciativa social y humanitaria en Europa Occidental. In: LORENZO GARCÍA R. DE; CABRA DE LUNA, M. A.; GIMÉNEZ-REYNA RODRÍGUEZ, E. Las entidades no lucrativas de carácter social y humanitario. Madrid: Ed. La Ley, 1991.

KUHN, T. La estructura de las revoluciones científicas. Madrid: Fondo de Cultura Económica de España, 2002.

LEVITT, T. The Third Sector-New Tactics for a Responsive Society. Nueva York: Ed. A Division of American Management Associations, 1973.

MARCUSE, H. L' Homme unidimensionel. Paris: Minuit, 1968. 
MINTZBERG, H. Mintzberg y la dirección. Madrid: Ed. Diaz de Santos 1991.

MYRDAL, G. Objetividad en la investigación social. México: Fondo de Cultura Económica, 1970.

MONZÓN, J. L. La economía social en España. CIRIECEspaña,n. 0, p. 19-29,1987.

.; DEFOURNY, J. Economía social. Entre economía capitalista y economía pública. Valencia: Ed. CIRIEC-España, 1992.

MOYANO ESTRADA, E. El concepto de capital social y su utilidad para el análisis de las dinámicas del desarrollo. Revista de Fomento Social, Córdoba, n. 56, p. 37-65, 2001.

SALAMON, L.; ANHEIER, H. K. In Search of the Nonprofit Sector I: the Question of Definitions. Working Paper n. 2 of The John Hopkins Comparative Nonprofit Sector Project 1992. [Publicado también en Voluntas, v. 3, n. 2, 1992, p. 125-152].

SWEDBERG, R. Major Traditions of Economic Sociology. Annual Review of Sociology, Palo Alto, CA, USA, n. 17, 1991,p. 251-276, 1991.

SWEDBERG, R.; SMELSER, N. Handbook of Economic Sociology. Princenton: Princenton University Press, 1994

VERNIS, A. et al. La gestión de las organizaciones no lucrativas. Barcelona: Ediciones Deusto,r 1998.

WEISBROD, B. A. Towards a Theory of the Voluntary Nonprofit Sector in a Three Sector Economy. In: PHELPS, E. (Ed.) Altruism, Morality and Economic Theory. New York: Russell Sage Foundation, 1975.

ZUBERO, I. Solidaridad. In: ARIÑO, A. Diccionario de la solidaridad (I). Valencia: Ed. Tirant lo Blanc, 2003, p. $463-$ 475.

\section{Notas}

1 La cuantificación de la economía social y el tercer sector ha sido tratada por diversos estudios tanto a nivel nacional como internacional. Para el conjunto de la Unión Europea es estiman 9 millones de empleos equivalentes a tiempo completo (el 6,6\% del empleo civil y el 8\% del empleo civil asalariado (CHAVES et al., 2000) y para el estado español (GARCÍADELGADO, 2004) del 6,5\% de entidades; el 6,9\% de asalariados y el 9,7\% de empleo equivalente (con voluntarios), es decir, en este último supuesto, 1,3 millones de puestos de trabajo; y un $4,7 \%$ de valor añadido bruto merece sin duda una atención especial.
2 El Comité Nacional deEnlace de las Actividades Mutualistas, Cooperativas y Asociativas (Cnlamca) aprobó en 1982 la Carta de la Economía Social que define a ésta como "el conjunto de entidades no pertenecientes al sector público que, con funcionamiento y gestión democráticos e igualdad de derechos y deberes de los socios, practican un régimen especial de propiedad y distribución de las ganancias, empleando los excedentes de ejercicio para el crecimiento de la entidad y la mejora de los servicios a los socios y a la sociedad" (MONZÓN, 1987). También en Bélgica, el Conseil Wallon de l'Économie Sociale (1990) estableciólas siguientes características para delimitar el sector de la Economía Social: finalidad de servicio a sus miembros o a la colectividad antes que de lucro; autonomía de gestión; procesos de decisión democráticos; primacía de las personas y del trabajo sobre el capital en el reparto de las rentas.

3 La denominación de entidades mutualistas y altruistas fue utilizada originariamente por Lord Beveridge, en un conocido informe de 1948, refiriéndose con el primer término a las entidades que satisfacen necesidades de sus miembros, y con el segundo a las que están dedicadas a la satisfacción de las necesidades de grupos más desfavorecidos (apud CABRADELUNA, 1998, p.31).

4 En el sector de mercado son muchas las diferentes clases de empresas que pueden ser incluidas en el ámbito de la economía social: cooperativas, sociedades laborales, sociedades agrarias de transformación, mutuas, mutualidades, empresas mercantiles controladas por la economía social e incluso cajas de ahorro. En el sector de no mercado, asociaciones y fundaciones constituirían las principales instituciones de la economía social. Esta definición no es unánime si nos referimos al caso español. Así Ballestero (1990) entiende que la economía social es aquel sector en donde no hay una división especializada de funciones entre el empresario y el trabajador. Bajo una estricta interpretación en esta definición se incluiría el trabajo autónomo pero se excluiría el fenómeno del movimiento asociativo organizado.

5 Como señala Myrdal (1970, p. 24), "Los hechos científicos no existen per se (por sí mismos), ni esperan para ello ser descubiertos por los científicos. Un hecho científico es una construcción abstraída de una realidad desarrollada y compleja con ayuda de fijaciones y clasificaciones arbitrarias. El éxito de los métodos para la elección de un problema y de una hipótesis fundamental, para la delimitación del ámbito de la investigación, y la definición de los datos relevantes del problema, significa que el investigador ha acertado en su elección. La elección ha sido un hallazgo efectuado a partir de un número ilimitado de posibilidades."

6 Kuhn (2002) puso de relieve que las comunidades científicas se constituyen por su adscripción a un determinado paradigma. No pretendemos que la afirmación realizada en el texto tenga, ni mucho menos, el alcance que a los paradigmas científicos les otorga Kuhn, pero sí nos parece que las 
cuestiones señaladas relativas a la delimitación del objeto de estudio constituyen un presupuesto, casi ineludible, para avanzar en la formación de una comunidad de investigadores.

7 De momento la Economía Social, al menos en España, ha tenido dos cauces para su desarrollo: estar presente en las líneas de investigación en las que trabaja el personal investigador de los departamentos universitarios, o estructurarse de manera interdepartamental a través de Institutos Universitarios de Investigación y de Grupos de Investigación, o a través de cátedras financiadas por instituciones privadas.

8 Es lo que Giddens (1997, p. 191-194) denomina doble hermenéutica: "El observador de ciencia social tiene que ser capaz primero de aprehender esos conceptos legos, o sea, penetrar hermenéuticamente en la forma de vida cuyas característicasquiere analizaroexplicar".Porello, "lainmersión en una forma de vida es el medio único y necesario. [...] Llegar a conocer una forma de vida es saber cómo orientarse en ella, ser capaz de participar en ella como un conjunto de prácticas. Pero para el observador sociológico este es un modo de generar descripciones que tienen que ser mediadas, o sea, transformadas en categorías del discurso de ciencia social“". En definitiva, reinterpreta los marcos de sentido de los actores sociales mismos dentro de sus esquemas teóricos mediante el lenguaje corriente y el técnico.

9 "La estructura aparece a la vez como condición y consecuencia de la producción de interacción" (GIDDENS, 1997,p. 189).

10 Es lo que Giddens (1997, p. 193) denomina "dualidad de estructura".

11 Si no se otorga una singularidad a estas realidades, los investigadores en el ámbito de la gestión de organizaciones y los directivos de estas entidades realizarán una translación casimimética de lo que ocurre en las empresas. Por el contrario, si se estima que estas entidades operan en un marco donde priman la cooperación y la solidaridad, olvidándose de las estructuras sociales en que deben actuar, se actuará en muchas ocasiones desde el voluntarismo más que desde la profesionalidad.

12 Como señala Herrera Gómez (1998, p. 28-29), se trata de una representación relacional del sistema societario, que concibe a éste a como un "retículo de redes relacionales' que tienden a diferenciarse entre ellas para cumplir determinados objetivos y funciones". Desde esta perspectiva, la sociedad es entendida como un sistema compuesto de cuatro subsistemas: "el mercado, entendido como el sistema de empresas que actúan por el beneficio; el Estado, caracterizado por el uso del instrumento del poder político (por tanto, del derecho) para el establecimiento forzoso de las obligaciones de ciudadanía y la redistribución de los recursos, el tercer sector, es decir, aquel conjunto de organizaciones autónomas no de beneficio que actúan motivadas por objetivos solidarios y que en primer término, aunque no exclusivamente, usan medios solidarios; $y$ las redes primarias: la familia, parientes, grupos de amigos y redes informales, que, en conjunto todos ellos constituyen un cuarto sistema en cuanto desempeñan funciones sociales insustituibles (sin equivalentes funcionales)."

13 Otra clave epistemológica es aquella que incide en sus aspectos económicos: como respuesta posible ante los 'fallos del mercado'. Puede verse el desarrollo de este enfoque en el trabajo de Ben-Nery Gui (2002).

14 Las afirmaciones anteriores no nos deben llevar a colegir que únicamente en el escenario social del tercer sector es en el que está presente la solidaridad. En el mundo de lo público, los valores de la justicia y de la solidaridad están omnipresentes - o al menos deberían estarlo-, pero se trata de una solidaridad administrativa y burocrática, en cuanto está reglada, y por ello es poco susceptible a recoger la dimensión interpersonal, con todo lo que esto comporta: las acciones del funcionario están reglamentadas en lo que se refiere a las necesidades que debe atender y la forma en que debe hacerlo. Asimismo, en el mundo del mercado, al menos en el de sus agentes, también inciden, en ocasiones, las motivaciones solidarias, ya sea como oferentes (mecenazgo empresarial, responsabilidad social de la empresa ...) o demandantes (campañas de presión a empresas en torno a productos muy competitivos pero producidos en condiciones inaceptables).

15 El mercado constituye, en última instancia, el espacio privilegiado para que opere el individuo "egoísta racional" (ZUBERO, 2003, p. 465), lo que excluye, en numerosas ocasiones, al valor de la solidaridad. Las tesis del egoísmo como guía de la acción encuentran sus precursores en Maquiavelo y Hobbes, quienes conciben al ser humano como fundamentalmente egoísta. "Esta visión deriva de la ubicación del hombre en el lugar central de la historia frente a la anterior visión teologizante dominante en el medievo. Esta centralidad del individuo dará lugar a los planteamientos liberales según los cuales la defensa del interés propio es entendida como orientadora de la acción social, lo que cristaliza en las prescripciones normativas que a partir de estaidea se desarrollan"(FUNES, 2003, p. 23).

16 Se ha señalado que la solidaridad administrativa ha resuelto algunos aspectos de la pobreza material, pero que ni puede ni le corresponde disponer de instrumentos para detectar la pobreza postmaterial y la precariedad de las relaciones interpersonales: la soledad, la desdicha, la desesperanza, la angustia, la incomunicación(VV.AA.ElEstado de Bienestar, 1996, apudCABRADELUNA, 1998,p. 19).Esteplanteamiento ha sido también destacado en 1991 por la Doctrina Social de la Iglesia en Centesimus annus, 48: "Efectivamente, parece que conoce mejor las necesidades y logra satisfacerlas de modo más adecuado quien está próximo a ellas o quien está 
cerca del necesitado. Además, un cierto tipo de necesidades requiere con frecuencia una respuesta que sea no sólo material, sino que sepa descubrir su exigencia humana más profunda, que requiere no sólo aportar los cuidados necesarios, sino un apoyo sinceramente fraterno" (atención a emigrantes, ancianos, enfermos, drogadictos etc.).

17 En el ordenamiento jurídico español, el cargo de patrono debe ejercerse necesariamente de forma gratuita (artículo 15.4 de la Ley 50/2002, de 26 de diciembre de Fundaciones). En el caso de las asociaciones, los miembros de los órganos de representación pueden percibir retribuciones con ciertas limitaciones (artículo 11.5 y 32.1 c) de laLey Orgánica 1/2002 de Asociaciones) En la práctica lo más frecuente es el desempeño gratuito del cargo, y, en cualquier caso, es indudable que la posibilidad de ausencia de retribución que se deduce del tenor de la propia Ley - constituye un cauce adecuado para el ejercicio de actividades solidarias, lo que no acontece en la entidades mercantiles, en las que por tradición, e incluso por exigencias jurídicas, resultaimpensable la gratuidad del cargo de miembro del consejo de administración.

18 Esto se constata en que muchos sujetos prefieren trabajar en estas entidades aun con retribuciones inferiores a las que obtendrían por desempeñar cargos similares en el sector público o en entidades mercantiles, o con un mayor grado de inestabilidad - la viabilidad económica de muchas de estas entidades no es comparable al de las entidades estrictamente capitalistas -, o incluso con una mayor exigencia de compromiso personal en lo cuantitativo - tiempo de trabajo - y en lo cualitativo - mayor implicación de aspectos no exclusivamente 'profesionales' del sujeto.

19 En esta línea, y de forma ilustrativa, afirma Zubero (2003,p. 463) que el movimiento voluntario debe ser "una compleja y preciosa máquina multiplicadora de solidaridad: el combustible del que se nutre es la solidaridad existente en el cuerpo social, solidaridad que devuelve a la sociedad purificaday multiplicada."

20 Como señala García Roca (1996, p. 33), la economía ha invadido numerosos territorios: "El poder de unificación que tenían las convicciones en la sociedad rural queda sustituido por la centralidad de la economía, que traspasa cuantas fronteras existen. De ningún modo puede despreciarse el poder de la economía para colonizar las relaciones sociales ni el imperio de la riqueza parainvadir la vida privada, las prácticas cotidianas y el imaginario social." Esta centralidad de la economía ha llevado a que muchas personas sólo sean capaces de pensar y actuar en una única dimensión (MARCUSE, 1968, p. 37): la del bienestar, el gasto y el consumo, dando lugar al denominado hombre unidimensional.

21 Esta es una de las funciones que asigna a las entidades sin ánimo de lucro el Consejo Económico y Social Francés en un informe de 1986 (apudCABRADELUNA, 1998, p.43-44).

22 Mintzberg (1991) se refiere a esta agilidad en estos términos: "Todo el mundo actúa de acuerdo con las creencias que lo impregnan todo, y pueden realizar el trabajo operativo con relativa independencia. Esto implica pocas normas y reglamentos formales en la organización misionera, poca planificación o control formal, ni siquiera mucho control directivo, y, por tanto, casi ninguna tecnoestructura ojerarquía de autoridad. Entonces, el liderazgo se convierte no tanto en la imposición de la dirección como en la protección y el enaltecimiento de la ideología común. Se espera que el líder inspire a otros para seguir la misión, quizás también que la interprete, pero nunca que la cambie."

23 En la producción de muchos servicios se requiere que el usuario participe en algún momento de su proceso de producción (educación, integración social, etc.) lo que provoca que el servicio prestado sea diferente en cada caso. En este sentido, los servicios, a diferencia de los bienes, tienen un carácter heterogéneo y no homogéneo, pues el elemento más importante son las personas que intervienen. Además, los servicios son producidos y consumidos, en gran medida, de forma simultánea (no cabe su almacenamiento o su adquisición para transportarlos y consumirlos en un momento posterior). Finalmente, un servicio no es un objeto corpóreo o tangible, susceptible de ser evaluado en su materialidad física, de ser poseído, ni de ser probado antes de ser prestado. Los servicios más bien son actividades o serie de actividades, recibiendo el usuario en muchas ocasiones exclusivamente ideas y sensaciones. Esto provoca que los servicios sean intangibles, y cuanto más intangibles son, mas importante es tener en cuenta todo lo que rodea a la prestación del servicio(VERNIS et al., 1998, p. 94-95).

24 Hansmann ha puesto de relieve la preferencia del consumidor por las ENL en los casos en que el mercado no es lo suficientemente transparente como para garantizar un control sobre la calidad del producto o del servicio ofertado mediante tradicionales contratos mercantiles. Desde este prisma, las ENL se presentan más competitivas en torno a la oferta de garantías, por cuanto instaurarían con el consumidor un 'contrato de confianza' fundado en el vínculo de la no distribución de los beneficios y, por consiguiente, en la falta de incentivos alaacumulación de los mismos (apud HERRERA GÓMEZ, 1998,p. 176).

25 En este sentido, compartimos plenamente la afirmación de que "una prestación sólo se convierte en tercer sector si es un servicio de proximidad, se sitúa en el interior de una comunidad humana con sus códigos simbólicos y expectativas personales"(GARCÍA ROCA, 1996, p. 21).

26 El capital social, en su acepción socioeconómica, se considera como una de las herramientas conceptuales más importantes para explicar las dinámicas de desarrollo. Moyano Estrada (2001) señala que fueron los economistas neoclásicos los 
que pusieron de manifiesto la importancia del capital humano considerando que sin dicho elemento, los demás factores trabajo, tierra y capital - tendrían poca utilidad para el desarrollo económico. Pero fueron los que trabajan en la nueva sociología económica (SWEDBERG, 1991, SWEDBERG; SMELSER, 1994) en su intento de explicarel comportamiento económico de los individuos, los que dieron contenido a dicho concepto englobando, no sólo las personas, sino también las normas y las redes sociales que contribuyen a la generación de acciones colectivas en beneficio de la propia comunidad.

27 La actividad en cuanto genere recursos y no se realice en condiciones inaceptables (corrupción, comisión de delitos) no será objeto de especial atención por parte de financiadores y trabajadores. Desde esta perspectiva, el objeto social más que un sentido aglutinador (lo que aglutina los intereses de los distintos miembros es la obtención de un lucro) puede tener un sentido disgregador si en la práctica se desarrolla a través de actuaciones en las que se comenten abusos: fraudes, corrupciones, explotación de trabajadores, discriminaciones.

28 En la práctica, se puede traducir en: a) que toda reducción de la actividad sea interpretada como una reducción del éxito de la organización (se pueden seguir desarrollando en el tiempo servicios ineficaces); b) que el servicio se 'presente' externamente como mucho más rico de lo que realmente es; c) que se produzcan bienes o servicios que son de hecho marginales o irrelevantes para sus beneficiarios, pero que los mecenas o donantes, ya sea por ignorancia o por otros motivos, desean financiar (HERRERA GÓMEZ, 1998,p. 191-192).

29 Sirva como ejemplo paradigmático el 'carisma' propio de muchas entidades religiosas, y la dificultad que tienen algunas de ellas para encontrar su auténtica razón de ser cuando han cambiado significativamente las necesidades sociales que se pretenden satisfacer respecto al momento fundacional del instituto religioso (promoción de la mujer a través de la educación, asistencia a menesterosos y enfermos).

\section{Dr. Julio Jiménez Escobar}

Doctor en Derecho

Profesor titular de la Facultad de Ciencias Económicas y Empresariales de Córdoba (ETEA)

\section{Dr. Alfonso Carlos Morales Gutiérrez}

Doctor en Ciencias Económicas y Empresariales Profesor titular de la Facultad de Ciencias Económicas y Empresariales de Córdoba (ETEA)

\footnotetext{
Facultad de Ciencias Económicas y Empresariales de Córdoba (ETEA)

Departamento de Economía General, Ciencias Jurídicas y Sociología
}

Estudios Cooperativas y Gestión de Entidades no Lucrativas (SEJ-148)

Calle Escritor Castilla Aguayo, 4

14004 Córdoba - España 